\title{
Notoginsenoside R1 Facilitated Wound Healing in High-Fat Diet/ Streptozotocin-Induced Diabetic Rats
}

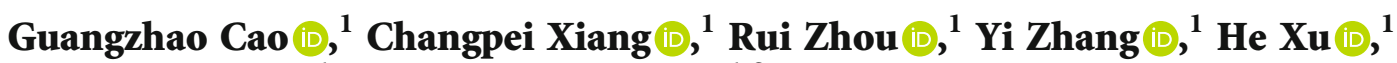 \\ Hongjun Yang $\mathbb{D}^{1}{ }^{1}$ and Jingjing Zhang $\mathbb{D i}^{1,2}$ \\ ${ }^{1}$ Institute of Chinese Materia Medica, China Academy of Chinese Medical Sciences, Beijing 100700, China \\ ${ }^{2}$ Experimental Research Center, China Academy of Chinese Medical Sciences, Beijing 100700, China \\ Correspondence should be addressed to Hongjun Yang; hongjun0420@vip.sina.com and Jingjing Zhang; jjzhang@icmm.ac.cn
}

Received 14 May 2021; Accepted 3 November 2021; Published 13 January 2022

Academic Editor: Jeferson Luis Franco

Copyright (c) 2022 Guangzhao Cao et al. This is an open access article distributed under the Creative Commons Attribution License, which permits unrestricted use, distribution, and reproduction in any medium, provided the original work is properly cited.

\begin{abstract}
Diabetic ulcers bring about high morbidity and mortality in patients and cause a great economic burden to society as a whole. Since existing treatments cannot fulfil patient requirements, it is urgent to find effective therapies. In this study, the wound healing effect of topical notoginsenoside R1 (NR1) treatment on diabetic full-thickness wounds in type II diabetes mellitus (T2DM) was induced by the combination of a high-fat diet and streptozotocin (STZ) injection. NR1 significantly increased the wound closure rate, enhanced extracellular matrix (ECM) secretion, promoted collagen growth, increased platelet endothelial cell adhesion molecule-1 (CD31) expression, and decreased cleaved caspase-3 expression. RNA-Seq analysis identified ECM remodeling and inflammation as critical biological processes and Timp1 and Mmp3 as important targets in NR1-mediated wound healing. Further experiments showed that NR1-treated wounds demonstrated higher expression of tissue inhibitor of metalloproteinase 1 (TIMP1) and transforming growth factor- $\beta 1$ (TGF $\beta 1$ ) and lower expression of matrix metallopeptidase 9 (MMP9), matrix metallopeptidase 3 (MMP3), interleukin-1 $\beta$ (IL-1 $\beta$ ), and interleukin-6 (IL-6) than diabetic wounds. These investigations promote the understanding of the mechanism of NR1-mediated diabetic wound healing and provide a promising therapeutic drug to enhance diabetic wound healing.
\end{abstract}

\section{Introduction}

Diabetic ulcers are serious complications that may lead to amputation in diabetic patients [1,2]. Insufficient blood supply, impaired angiogenesis, and associated infection have been identified as the major reasons to ulcers in diabetic patients [3]. Studies have shown that nearly $25 \%$ of diabetic patients will progress to diabetic ulcers, which seriously threatens their health [4]. Wound healing in diabetes is associated with a series of pathological processes [5], such as oxidative stress [6], persistent inflammation [7], and apoptosis [8], and a decrease in extracellular matrix (ECM) secretion, resulting in nonunion of diabetic wounds [9]. Furthermore, macrophages and neutrophilic granulocytes aggregate slowly in the persistent inflammatory stage and correspond to increased inflammation in diabetic ulcer patients $[10,11]$, which is different from the inflammatory stage of normal wounds. At this time, the phagocytosis ability of leucocytes is impaired, and the existence time of inflammatory cytokines in wounds is prolonged, which inhibits the formation of granulation tissue and wound healing [12]. Meanwhile, abnormal cell apoptosis further prevents normal wound healing [8]. In addition, insufficient angiogenesis is a key factor in delayed diabetic wound healing $[5,13]$. The ECM is expressed during wound healing and is essential for tissue repair, and its abnormality mainly affects the remodeling and proliferation stages of wound healing [9].

Current treatments include debridement [14], wound dressings [15], negative pressure wound therapy (NPWT) [16], and epidermal growth factor (EGF) [17]. However, there are some limitations to these treatments. For example, debridement often requires local infiltration with a significant risk of complications [18]. NPWT is a major clinical 
treatment, but the good results of NPWT can only be achieved when using a debridement to remove all devitalized, necrotic, and infected tissues [19]. The application of wound dressings depends on the type and severity of the wounds, as well as the condition of the patients [20]. Above all, these treatments are still unable to fulfill the patients' requirements and remain a huge challenge for diabetic wound healing. Therefore, it is important to find effective and convenient therapeutic strategies for diabetic wounds.

Recently, increasing evidence has demonstrated that traditional Chinese medicine (TCM) is effective and convenient for diabetic ulcer treatments. Our previous research indicated that topical treatment of the Huangbai line enhanced the key antioxidant regulator $\mathrm{Nrf} 2$ and its downstream antioxidant target genes, which increased wound closure and the synthesis of ECM and collagen while decreasing cell apoptosis and oxidative damage, accelerating wound healing in diabetes. As a major bioactive component from Coptis Salisb. (rhizome), berberine promoted wound healing in both type I diabetes and type II diabetes, which decreased oxidative damage, cell apoptosis, and ECM remodeling through thioredoxin reductase $1 / \mathrm{c}$-Jun $\mathrm{N}$-terminal kinase (TrxR1/JNK) signaling $[21,22]$. Panax notoginseng and its main bioactive components exhibit pharmacological activity in various diseases, such as cancer, hypoglycemic effects, neuroprotection, and diabetes complications [23-25]. As one of the most important active components extracted from Panax notoginseng Notoginsenoside R1 (NR1) [26], it demonstrated an effective effect on various diabetic complications including diabetic nephropathy [27] and diabetic encephalopathy [28]. Relevant studies have reported that Panax Notoginseng Saponins (PNS) can alleviate skin ulcers in diabetic rats by reducing the expression of endothelin-1 (ET-1) and tumor necrosis factor- $\alpha$ (TNF- $\alpha)[29,30]$. However, the effect of NR1 on diabetic ulcers has not been reported, and the mechanism of NR-mediated wound healing remains unknown. In this study, the effect of topical NR1 treatment on type II diabetic wounds established by a high-fat diet and streptozotocin (STZ) injection combined with full-thickness wounds was systematically investigated by using RNA Sequencing (RNA-Seq) technology. Topical treatment of NR1 provides a convenient way to accelerate wound healing and allow NR1 to concentrate to the local wound site and avoid vascular dysfunction-induced absorption problems, offering a new treatment for diabetic ulcers.

\section{Materials and Methods}

2.1. Animals and Model Establishment. Sprague-Dawley rats that were adult male rats with weights between 160 and $180 \mathrm{~g}$ were supplied by the Beijing Huafukang Biotechnology Co., Ltd. (license number: SCXK (BJ) 2014-0004). All procedures in the experiment were conducted following the animal care and rules of the Institute of Chinese Materia Medica, China Academy of Chinese Medical Sciences. These rats were housed in an animal room with a 12-hour light/ dark cycle environment and access to food and water continued to be freely available for 3 days. After adaptive feeding for 3 days, rats had free access to water and a high-fat diet
TABle 1: Composition of the experimental high-fat diet.

\begin{tabular}{lc}
\hline Components & Mass ratio $(\mathrm{g})$ \\
\hline Protein (casein, L-cysteine) & 230 \\
Carbohydrate (dextrin, source) & 330 \\
Fat (soybean oil, lard) & 367 \\
Mineral and vitamin mixture & 73 \\
Antioxidant (TBHQ) & 0.07 \\
Energy (kcal/g) & 5.5 \\
$\%$ kcal from protein & $15 \%$ \\
Carbohydrate & $25 \%$ \\
Fat & $60 \%$ \\
Total & $100 \%$ \\
\hline
\end{tabular}

for 4 weeks. The composition of high-fat diet is shown in Table 1. Streptozotocin (STZ, $35 \mathrm{mg} / \mathrm{kg}$ ) was injected intraperitoneally into rats to induce diabetes and fasting for $12 \mathrm{~h}$ before the establishment of the diabetic model. For STZ-induced diabetes, the fasting blood glucose levels of rats randomly higher than $16.7 \mathrm{mmol} / \mathrm{L}$ but lower than $33.3 \mathrm{mmol} / \mathrm{L}$ were supposed to be diabetes. The diabetic rats were anesthetized with sodium pentobarbital, and two rubber rings (diameter, $2 \mathrm{~cm}$ ) were sutured onto the skin of the diabetic rats. Then, two wounds that were $2 \mathrm{~cm}$ in diameter were symmetrically created on the rats' dorsum, with the left side rubbed by physiological saline and the right side rubbed by drugs [31]. Diabetic rats were divided into three groups according to a random number, and the rats received different treatments: physiological saline (hfdSTZ), NR1 (hfdSTZ+NR1, $0.038 \mathrm{mg} / \mathrm{cm}^{2}$ ), and rhEGF (hfdSTZ+rhEGF, $40 \mathrm{IU} / \mathrm{cm}^{2}$ ). All rats received topical therapy once per day for 15 consecutive days. After 14 days of treatment, fasting blood glucose levels were measured again. It is also noteworthy that in our experiment, the rats with fasting blood glucose levels between $16.7 \mathrm{mmol} / \mathrm{L}$ and $33.3 \mathrm{mmol} / \mathrm{L}$ were included. Representative photos of wound areas were taken at $0,6,9,12$, and 15 days during the treatment, and the wound closure was measured using ImageJ software, after which the wound closure rate was calculated.

2.2. Two-Photon Microscope- (SHG-) Based Collagen Growth Observation. First, collagen growth at the edge of the wounds was investigated in the rats by a two-photon microscope (SHG) after anesthetizing them with sodium pentobarbital.

2.3. Hematoxylin and Eosin (HE) Staining and Masson's Trichrome Staining. The skin tissues were placed into a new $4 \%$ paraformaldehyde solution at room temperature for 3 days. Then, the paraffin-embedded tissues were cut into sections with a thickness of $6 \mu \mathrm{m}$ for HE and Masson's trichrome staining. The stained slide was observed using a panoramic Digital Slide Scanner.

2.4. RNA-Seq Analysis. RNA extraction from the skin tissue was conducted with TRIzol reagent, and its integrality was measured. After quantification, RNA was then used to 
construct cDNA libraries. The sequencing process was completed according to previous protocols on an Illumina HiSeq 4000 platform, which was finished by Novogene Bioinformatics Technology Co., Ltd. (Beijing, China). Raw data for NR1-mediated wounds have been uploaded to the https://www.ncbi.nlm.nih.gov/sra/PRJNA757790. Differentially expressed genes (DEs) were also defined by using $\log 2$-fold change and $p$ value analysis through the EBSeq algorithm, followed by a volcano plot to show the overall distribution. Then, genes with different expression patterns were grouped by hierarchical clustering. GO and KEGG enrichment analyses of DEs were performed using the DAVID, and the items were considered significant if the $p$ value was less than 0.05 . The biological processes and pathways associated with diabetic ulcers are shown in the bubble chart. Finally, a network of DEs and the enriched GO terms was then constructed via STRING and Cytoscape software.

2.5. Enzyme-Linked Immunosorbent Assay (ELISA). Skin tissue homogenate was prepared for the following measurements. Levels of interleukin-1 $\beta$ (IL- $1 \beta)$, matrix metallopeptidase 9 (MMP9), interleukin-6 (IL-6), transforming growth factor- $\beta 1$ (TGF- $\beta 1$ ), matrix metallopeptidase 3 (MMP3), and tissue inhibitor of metalloproteinase 1 (TIMP1) were measured by ELISA. All steps were performed according to the manufacturer's instructions. Briefly, the sample was added to the ELISA plate coated with the monoclonal antibody and incubated for $1 \mathrm{~h}$. Then, the biotinylated-labeled secondary antibody was added to the plate for $1 \mathrm{~h}$. Next, the TMB substrate solution was added to the plate for $20 \mathrm{~min}$ before stopping the reaction with the stop solution. Then, the absorbance value was measured with a microplate reader (Molecular Devices, USA) at $450 \mathrm{~nm}$.

2.6. Immunohistochemistry. Briefly, the skin tissues were rehydrated with $\mathrm{H}_{2} \mathrm{O}_{2}$ after dewaxing. Sections were loaded with the primary antibodies overnight. Secondary antibody incubation was followed by incubation for $10 \mathrm{~min}$ with streptavidin peroxidase. DAB (1x) was applied as the chromogen to incubate with the sections for $10 \mathrm{~min}$ at room temperature and then dyed by hematoxylin counterstain. Photos were captured under a light microscope and counted using ImageJ software. The antibodies used were as follows: TGF- $\beta 1$ (sc-130348, Santa), MMP9 (ab76003, Abcam), platelet endothelial cell adhesion molecule-1 (CD31) (A0378, Abclonal), and cleaved caspase-3 (9664S, CST 9664S).

2.7. Statistical Analysis. Differences in data obtained in our manuscript were analyzed by one-way ANOVA, and all values are expressed as the mean \pm S.D. $p<0.05$ means that the difference between tested groups is statistically significant.

\section{Results}

3.1. Topical NR1 Treatment Enhanced the Healing of Diabetic Wounds. To investigate the effect of NR1 on diabetic wounds, the wound closure rate was evaluated. The hdfSTZ group showed a lower wound closure rate than the control group (Figures 1(b) and 1(c)). NR1 significantly increased the wound closure rate at Days 6, 9, 12, and 15 compared to the hfdSTZ group, which was equivalent to that in the hfdSTZ+rhEGF group. Thus, topical NR1 and rhEGF treatment enhanced wound healing in T2DM.

3.2. NR1 Decreased Cleaved Caspase-3 and Increased ECM Secretion and CD31 Expression in Diabetic Wounds. To investigate the effect of NR1 on ECM secretion, apoptosis and angiogenesis in diabetic ulcer rats, HE staining, cleaved caspase-3, and CD31 expression were evaluated. The results revealed that the epithelial tissue (black arrow in Figures 2(a) and 2(c)) in the diabetic wounds was significantly increased after NR1 or rhEGF treatment, whereas there was no significant repair in the hfdSTZ group (yellow arrow in Figure 2(a)). This further proved that re-epithelialization was accelerated in the hfdSTZ+NR1 group and hfdSTZ+rhEGF group. In addition, there was more blood capillary regeneration (black triangle in Figures $2(\mathrm{a})$ and $2(\mathrm{~d})$ ) in the hfdSTZ+NR1 group and hfdSTZ+rhEGF group than in the hfdSTZ group. Furthermore, there was lower secretion of ECM in the hfdSTZ group than in the control group at Day 15 , but the secretion of ECM was significantly increased after treatment with NR1 and rhEGF (Figures 2(a) and 2(e)). In addition, as shown in Figures $2(\mathrm{f})$ and $2(\mathrm{~g})$, an obvious increase in cleaved caspase- 3 and a decrease in CD31 were observed in the hfdSTZ group compared to the control group, whereas NR1- and rhEGF-treated wounds displayed lower cleaved caspase- 3 expression and higher CD31 expression. The immunochemical staining results identified that cleaved caspase-3 expression in the hfdSTZ+NR1 group and hfdSTZ+rhEGF group was lower than that in the hfdSTZ group, and CD31 staining was higher than that in the hfdSTZ group (Figure 2(b)). Thus, NR1 and rhEGF reduced apoptosis and promoted angiogenesis and ECM secretion in diabetic wounds.

3.3. RNA Sequencing to Identify the Possible Mechanism of NR1 in Diabetic Wounds. Compared to the control group, 809 DEGs were observed in the hfdSTZ group, with 181 upregulated genes and 628 downregulated genes. In contrast to the hfdSTZ group, 316 DEGs were significantly upregulated, and 167 genes were significantly downregulated in the hfdSTZ+NR1 group (Figure 3(a)). In addition, heatmap results indicated that the gene expression pattern was different between the hfdSTZ group and the NR1 group. The gene expression patterns of the hfdSTZ+NR1 group and the control group were similar (Figure 3(b)). To further understand the underlying biological processes and pathways, GO and KEGG enrichment analysis of DEGs between the hfdSTZ+NR1 and hfdSTZ groups was carried out (Figure 3(c)). Furthermore, these DEGs after NR1 treatment were enriched in biological processes and pathways such as the inflammatory response, response to hypoxia, chemotaxis, chemokine-mediated signaling pathways, extracellular matrix organization, TNF signaling pathway, tight junctions, and cell adhesion molecules.

3.4. Identification of Critical Biological Processes and Targets in NR1-Mediated Diabetic Wound Repair. A network of DEs and biological processes regulated by NR1, which is 


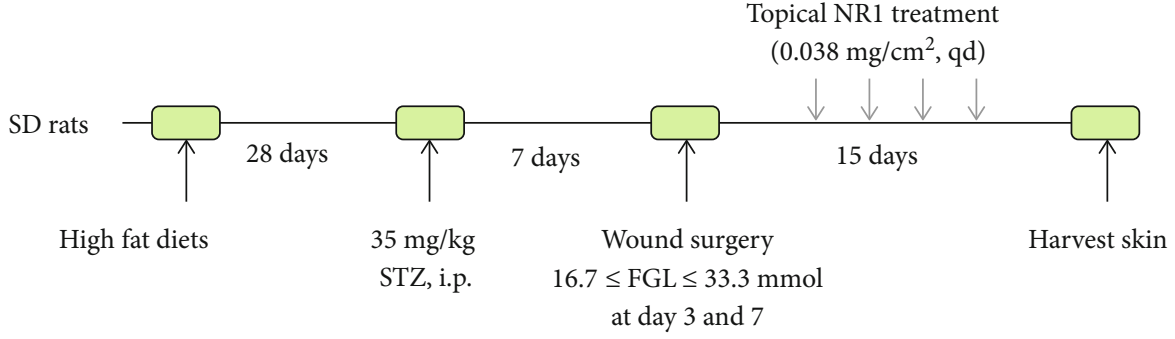

(a)
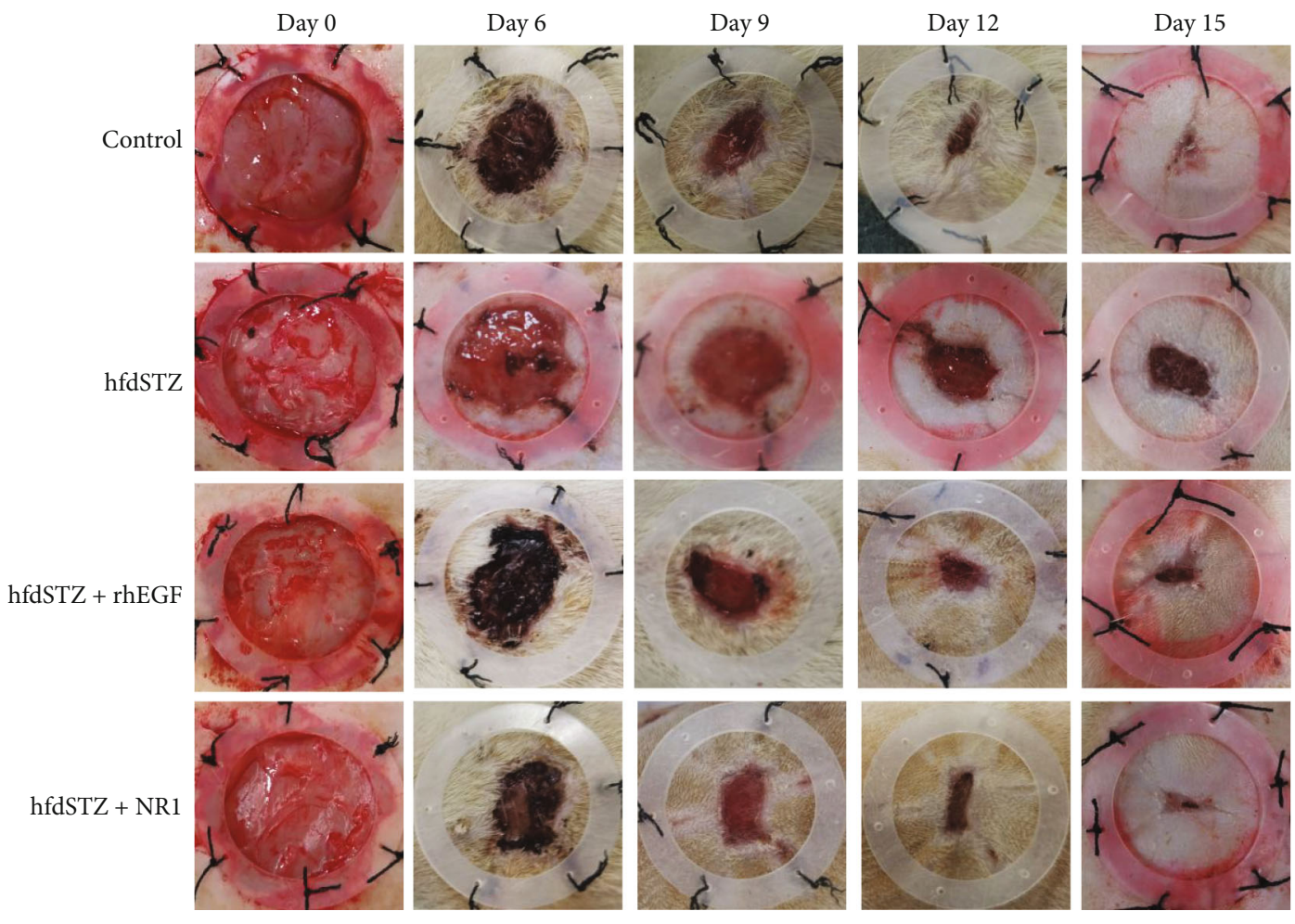

(b)

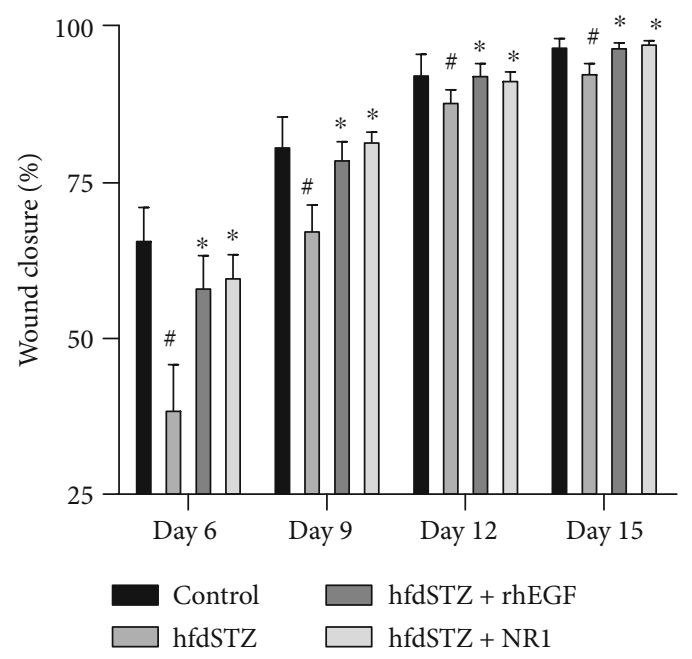

(c)

FIGURE 1: Topical NR1 treatment significantly promoted the wound closure rate in diabetic rats. (a) Schematic illustration showing the establishment of the diabetic ulcer model and treatment with NR1 for 15 days. (b) Representative images of diabetic wounds at Days 6, 9,12 , and 15 . (c) The wound closure rate was quantitated by ImageJ software $(n=7-10)$. Data are presented as the mean \pm SD; ${ }^{*} p<0.05$ vs. control; ${ }^{*} p<0.05$ vs. hfdSTZ. 
Control

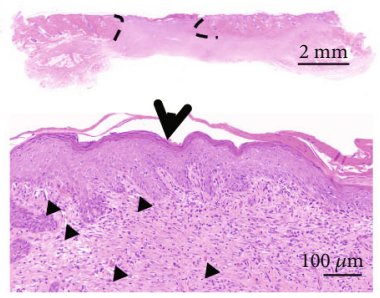

Control

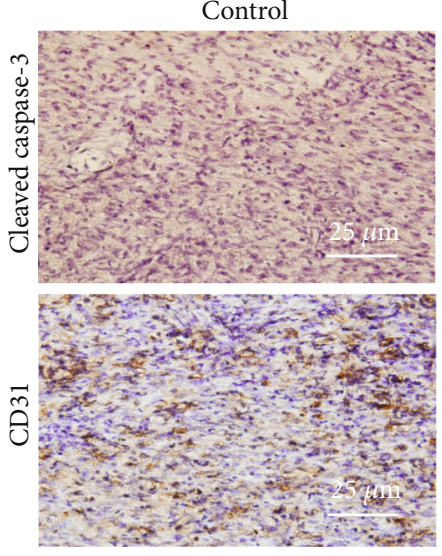

hfdSTZ
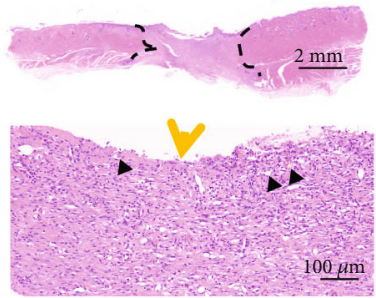

(a)
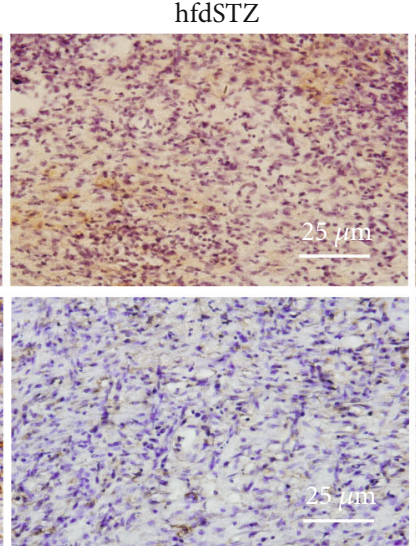

(b)

)

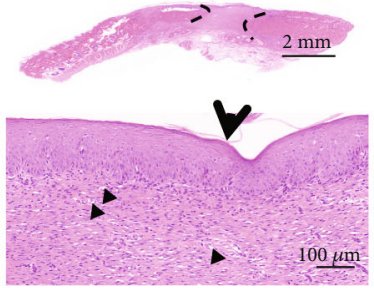

hfdSTZ+rhEGF
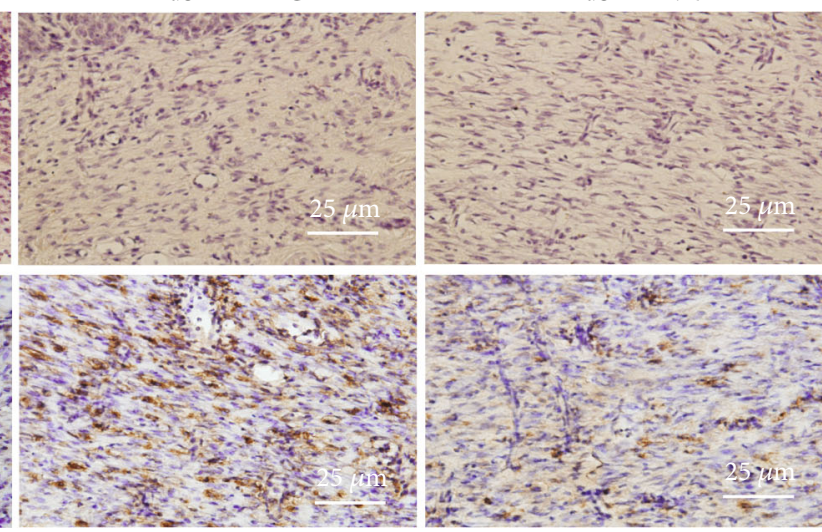

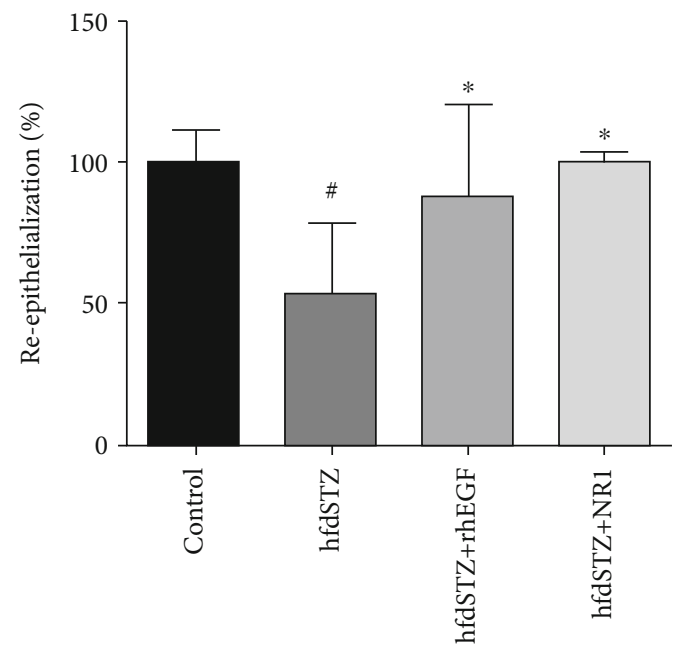

(c)

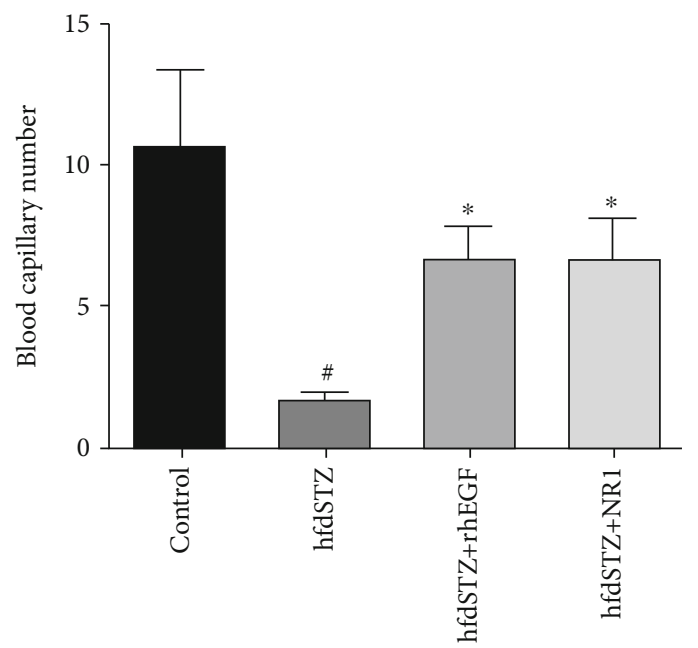

(d)

Figure 2: Continued. 


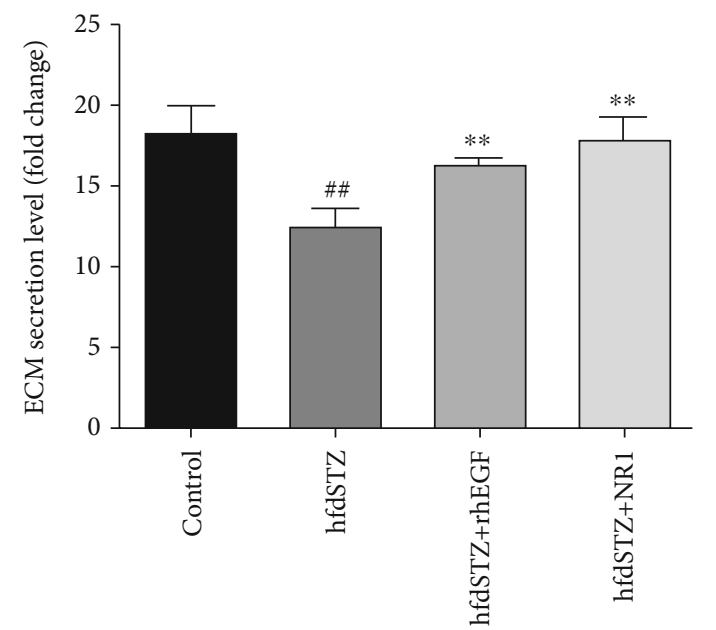

(e)

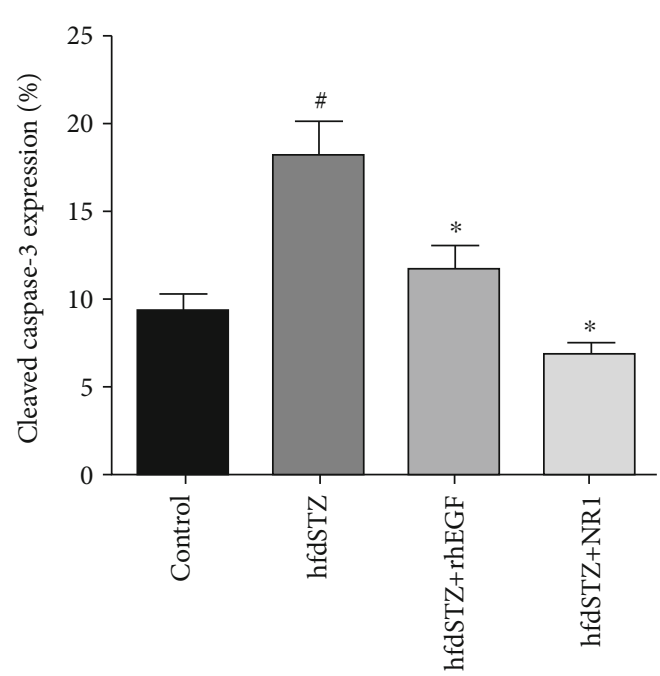

(f)

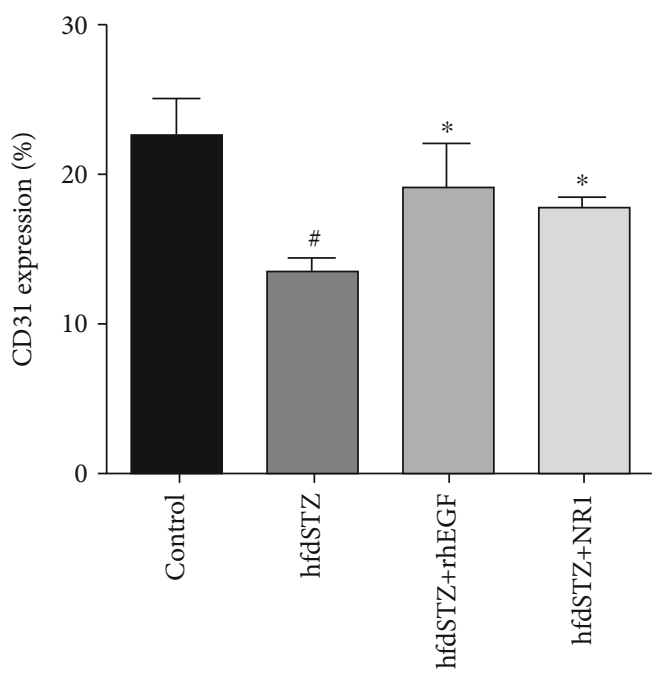

$(\mathrm{g})$

FIGURE 2: Effect of NR1 treatment on ECM secretion and the expression of cleaved caspase-3 and CD31 in diabetic wounds. (a) HE staining. (b) Immunohistochemical staining for cleaved caspase-3 $(n=4)$. Scale bar: $25 \mu \mathrm{m}$; immunohistochemical staining for CD31 $(n=3-4)$. Scale bar: $25 \mu \mathrm{m}$. (c) Quantified re-epithelialization $(n=3)$. (d) Quantified blood capillary $(n=3)$. (e) Quantified ECM secretion level $(n=3)$. (f) Quantified immunohistochemical staining of cleaved caspase-3 $(n=4)$. (g) Quantified immunohistochemical staining of CD31 $(n=3-4)$. Data are presented as the mean \pm SD. Significance: ${ }^{*} p<0.05$ vs. control and ${ }^{*} p<0.05$ vs. hfdSTZ.

associated with diabetic wound repair, was constructed. As shown in Figure 4, we identified ECM-related processes and inflammation as critical processes, since the DESs were enriched in GO terms such as extracellular matrix disassembly, extracellular matrix organization, immune response, inflammatory response, chemotaxis, and chemokine-mediated signaling pathway. By analyzing the degree of the targets in this network, targets, such as Cxcl1, Fos, Timp1, and Mmp3, with the highest degree were identified as vital targets in NR1mediated wound healing.

3.5. Verification of the Critical Biological Processes and Targets in NR1-Mediated Diabetic Wound Healing. To verify the critical biological processes, ECM synthesis and remodeling were evaluated by determining collagen growth and the expression of MMP9 and TGF- $\beta 1$. Collagen synthesis was visualized using a two-photon microscope (SHG) and Masson's staining. The SHG results demonstrated that the collagen expression at the front edge of wounds was lower at Day 15 in hfdSTZ than in the control, whereas this was increased after topical treatment with NR1 and rhEGF (Figure 5(a)). Consistent with this result, Masson's trichrome staining showed that NR1 and rhEGF treatment increased the low collagen expression in diabetic wounds (Figure 5(b)). The immunochemical staining and ELISA results showed that NR1 increased TGF- $\beta 1$ expression while decreasing MMP9 expression in diabetic wounds (Figures 5(c)-5(e), 6(a), and 6(b)). Taken together, NR1 promoted collagen expression and maintained ECM synthesis and remodeling in diabetic ulcer rats.

Since the DEGs after NR1 treatment were enriched in biological processes, such as inflammation, IL- $1 \beta$ and IL- 6 were measured. The ELISA results demonstrated that NR1- 

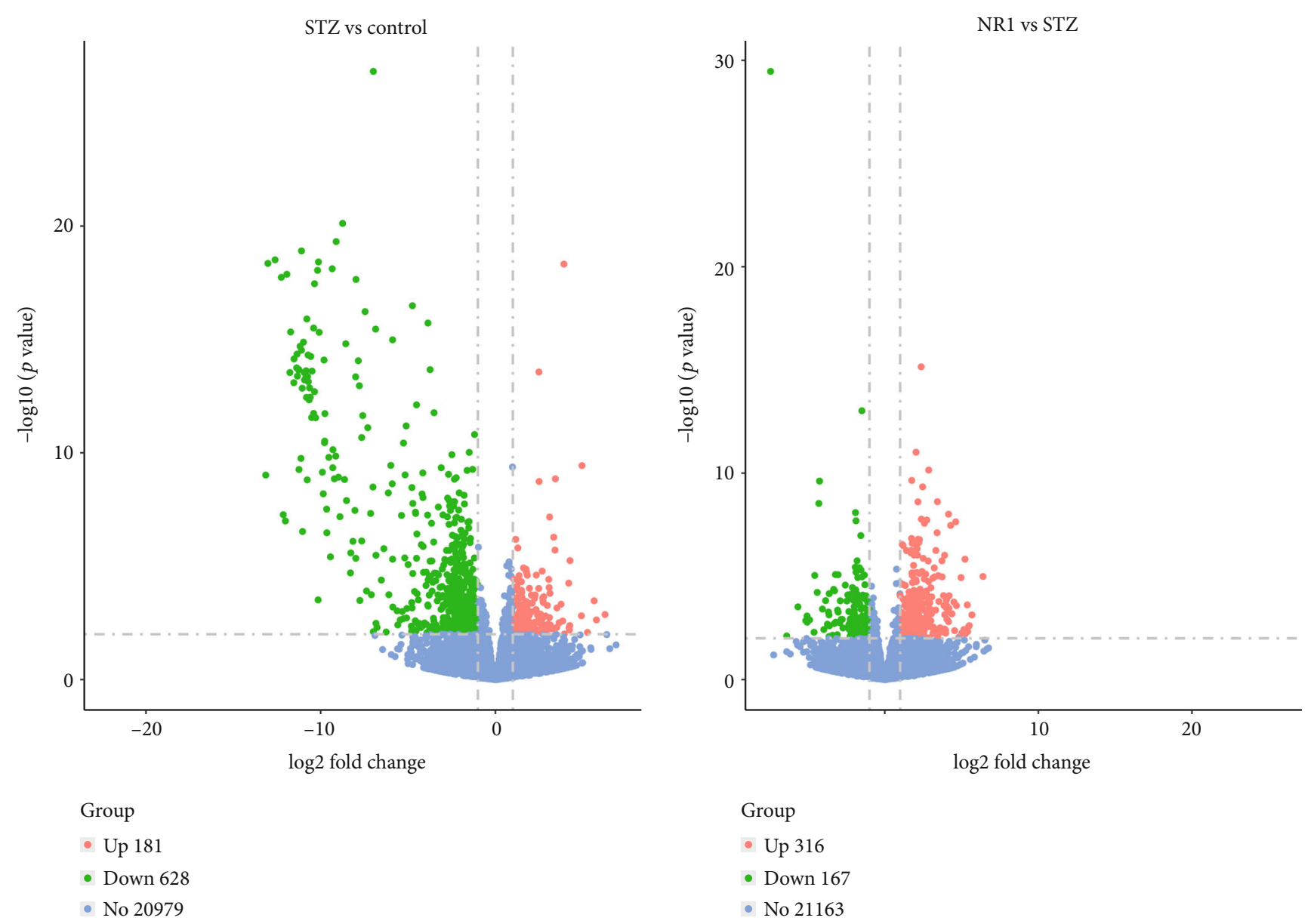

Group

- Up 181

- Down 628

- No 20979

(a)

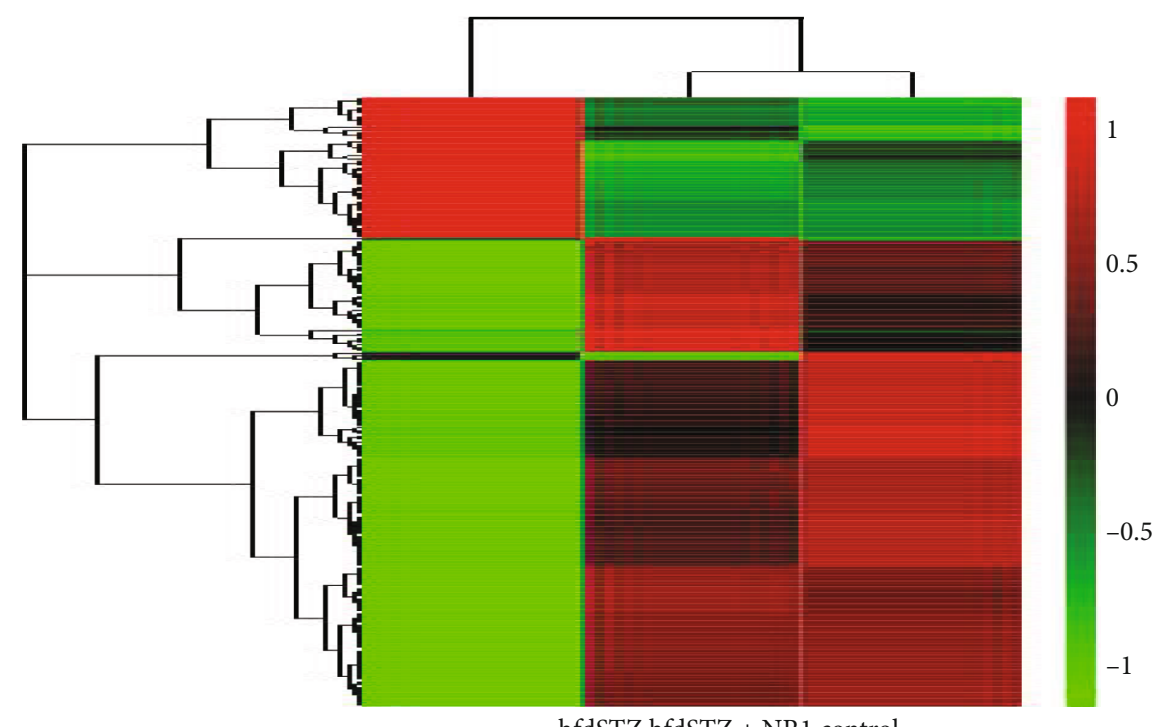

hfdSTZ hfdSTZ + NR1 control

(b)

Figure 3: Continued. 


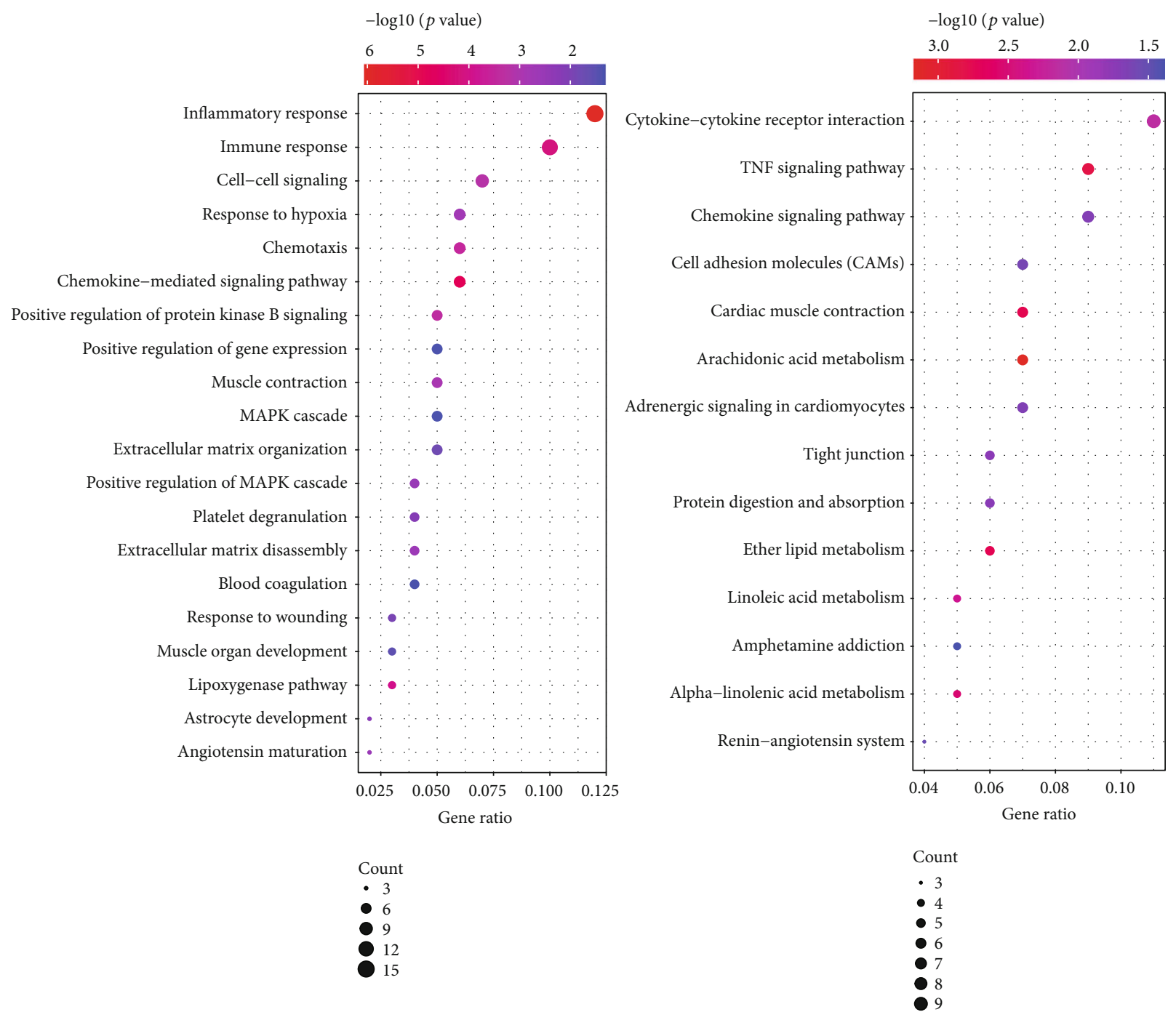

(c)

FIGURE 3: The global gene expression pattern of skin wounds. (a) Volcano map of the DEGs with upregulated genes (red) and downregulated genes (green); blue represents nondifferentially expressed genes. (b) The heatmap. (c) GO and KEGG pathway analysis of these DEGs; the horizontal axis represents the ratio of the number of differential genes and the total differential genes enriched in GO enrichment or KEGG pathway; the vertical axis represents GO or KEGG terms.

treated wounds had lower expression of IL- $1 \beta$ and IL-6 than hfdSTZ-treated wounds (Figures 6(c) and 6(d)). The identified critical targets, such as tissue inhibitor of metalloproteinase 1 (TIMP1) and MMP3, were also confirmed by ELISA. As shown in Figures 6(e) and 6(f), the high level of MMP3 in the hfdSTZ group was significantly reduced by NR1 and rhEGF treatment. In addition, a low level of TIMP1 in diabetic wounds was increased by NR1 treatment compared with that in the hfdSTZ group. Thus, NR1 reduced inflammation and ECM degradation while enhancing collagen secretion, and TIMP1 and MMP3 were potential critical targets.

\section{Discussion}

In this study, a skin ulcer model was established by fullthickness wounds in type 2 diabetic rats to evaluate the effect of NR1 on diabetic wound healing. Furthermore, the possi- ble mechanism of NR1 against diabetic ulcers was elucidated by using RNA-Seq technology. NR1 obviously enhanced the wound healing rate, promoted collagen expression, and increased CD31 expression while decreasing cleaved caspase-3 expression. By applying RNA-Seq technology, we identified extracellular matrix related processes and inflammation as critical biological processes and Timp1 and Mmp3 as important targets in NR1-mediated wound healing. Our further experiment confirmed the inhibitory effect of NR1 on ECM remodeling and inflammation, which was evidenced by low expression of MMP9, MMP3, IL-1 $\beta$, and IL- 6 and higher expression of TGF- $\beta 1$ and TIMP1.

Numerous studies have indicated that wound healing in diabetes is due to apoptosis [32] and insufficient angiogenesis $[5,13]$. In our study, we showed that NR1 decreased cleaved caspase- 3 and increased CD31 expression in diabetic wounds, which indicated that topical NR1 treatment 


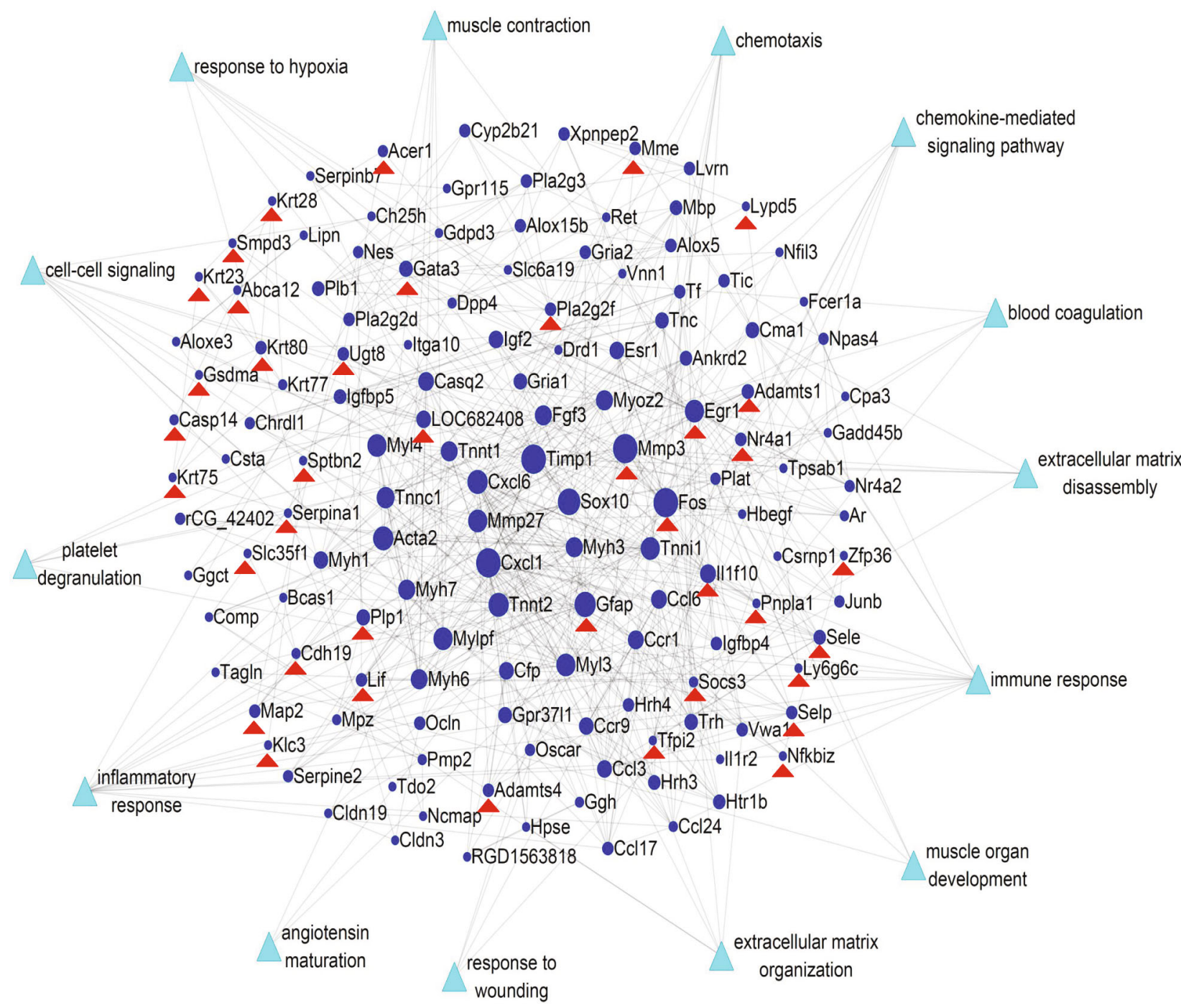

FIGURE 4: The network based on DEGs between the hfdSTZ group and hfdSTZ+NR1 group and biological processes associated with diabetic wound repair; red triangles represent DEGs in the hfdSTZ+NR1 group and hfdSTZ group; light blue triangles represent the biological processes associated with wound repair in diabetes.

attenuated apoptosis, increased angiogenesis, and consequently enhanced wound healing in diabetic rats. Angiogenesis is a key factor in facilitating wound healing [33, 34]. Abnormal angiogenesis can result in impaired wound healing, and the mechanism may be related to the change in macrophage phenotype and failure to promote tissue repair [13]. CD31 was previously widely applied as an angiogenesis marker $[35,36]$. Furthermore, abnormal apoptosis is associated with impaired wound healing, resulting in slow wound healing [8]. During the process of wound healing, apoptosis usually helps to remove inflammatory cells and is closely associated with the development of granulation tissue to scar tissue [37]. However, the accumulation of advanced glycation end products and the increase in ROS production caused mitochondrial damage, decreased expression of B-cell lymphoma2 (Bcl-2), and increased expression of caspase in diabetic wounds. Therefore, the proliferation of fibroblasts is inhibited, and wound healing is delayed [8]. Cleaved caspase- 3 acts as a major executive caspase in apoptosis, and a relevant study found that cleaved caspase- 3 activity is elevated in diabetic ulcer rats $[22,38-40]$. These results reflected our results that diabetic ulcers were associated with the biological processes of apoptosis and angiogenesis.

Importantly, inflammation is relevant for wound repair and leads to decreased vascularization and tissue necrosis, which impair wound healing $[41,42]$. IL- $1 \beta$, a proinflammatory cytokine, is considered an indicator of inflammation and plays an important role in the impairment of chronic and nonhealing diabetic wounds [43-45]. A previous study showed that the wound healing rate can be accelerated through the marked downregulation of IL- $1 \beta$ in diabetic foot ulcer rats [46]. IL-6 is another inflammatory cytokine that is thought to be related to wound healing and a relevant study showed that the production of IL-6 in dermal tissue delayed the dermal wound healing response [47]. Furthermore, longterm diabetic ulcers are usually accompanied by less ECM regeneration and excessive degradation, which is characterized by low TGF- $\beta 1$ and TIMP1 expression and high MMP3 and 
Control

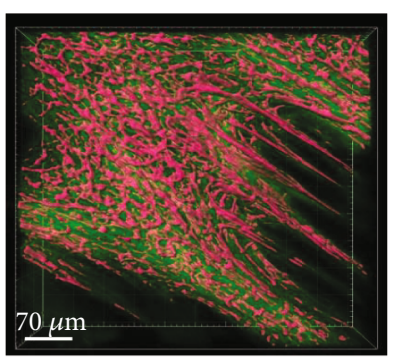

Contro
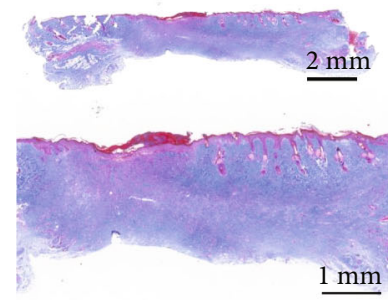

$\mathrm{mm}$
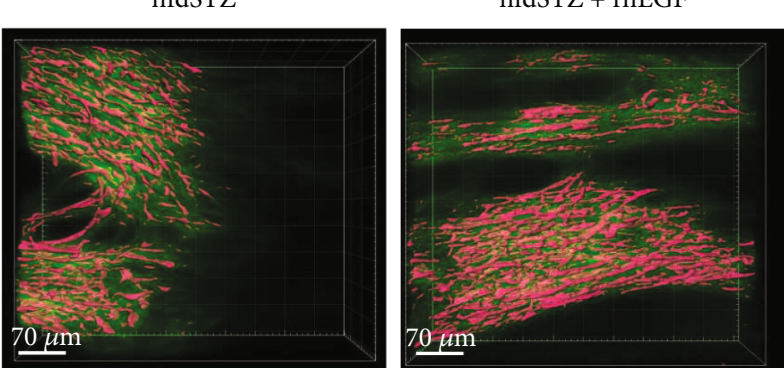

(a)
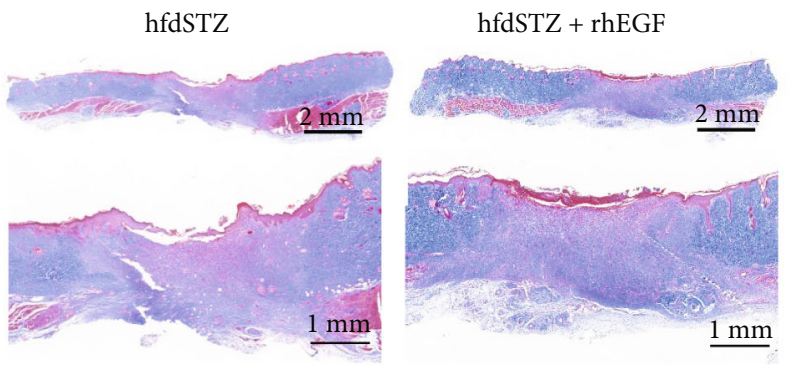

(b)
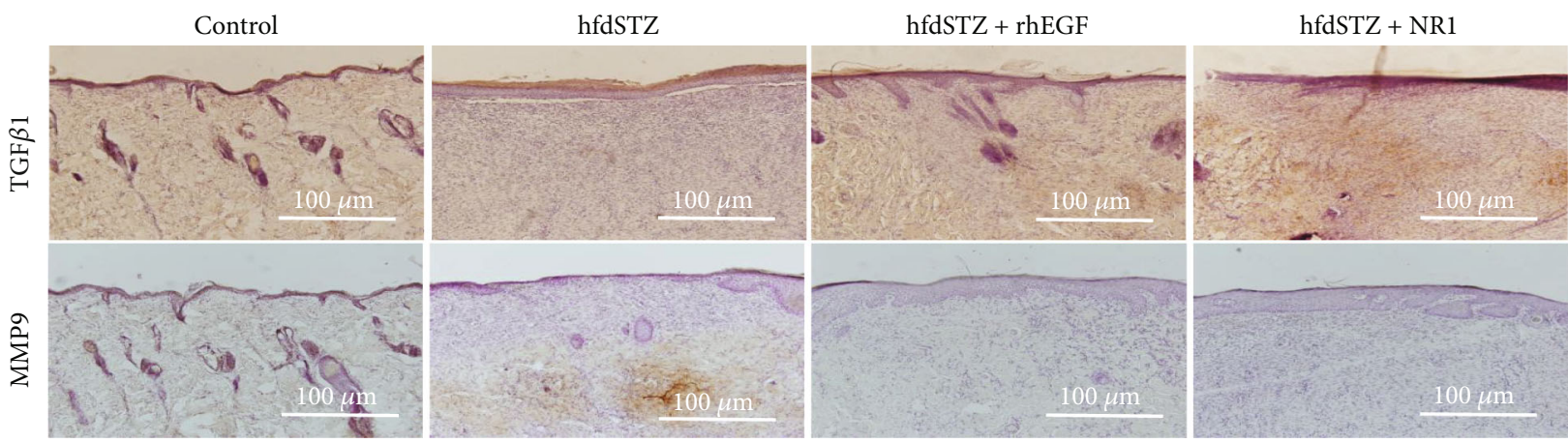

(c)

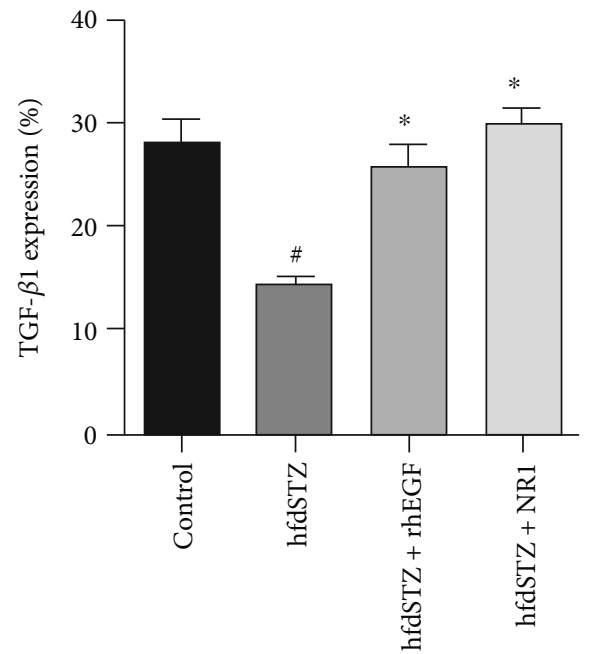

(d)

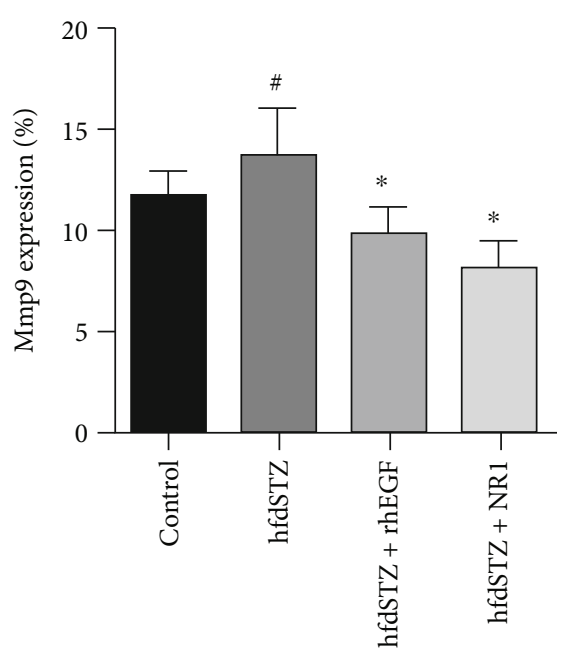

(e)

FIGURE 5: Topical NR1 treatment promoted collagen expression and maintained the generation and remodeling of ECM in diabetic rats. (a) Pink represents new collagen growth at the edge of diabetic wounds; green represents the background of Day 15 . Scale bar: $70 \mu \mathrm{m}$. (b) Masson staining. (c) Immunohistochemical staining for TGF- $\beta 1$ and MMP9 $(n=4)$. Scale bar: $100 \mu \mathrm{m}$; (d) Quantified immunohistochemical staining of TGF- $\beta 1(n=4)$. (e) Quantified immunohistochemical staining of MMP9 $(n=3)(n=4)$. Data are presented as the mean \pm SD. Significance: ${ }^{*} p<0.05$ vs. control and ${ }^{*} p<0.05$ vs. hfdSTZ. 


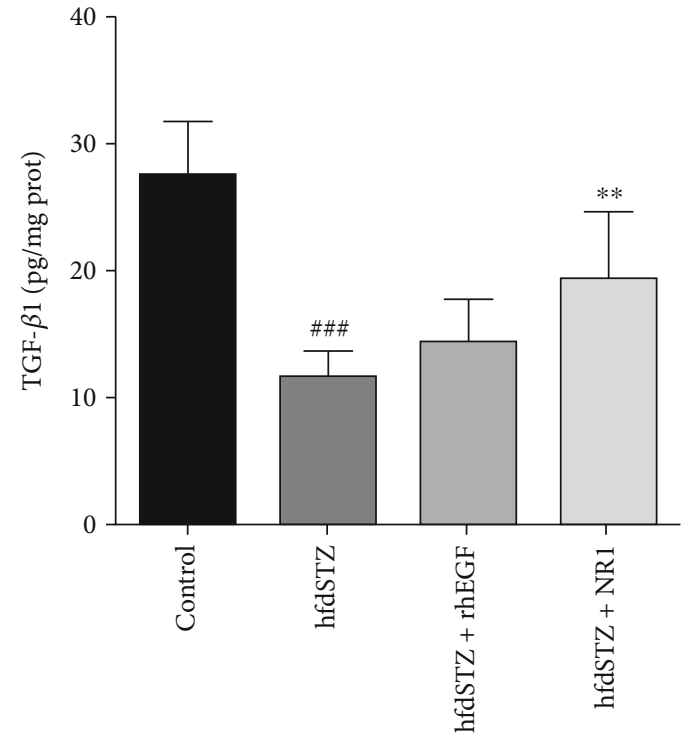

(a)

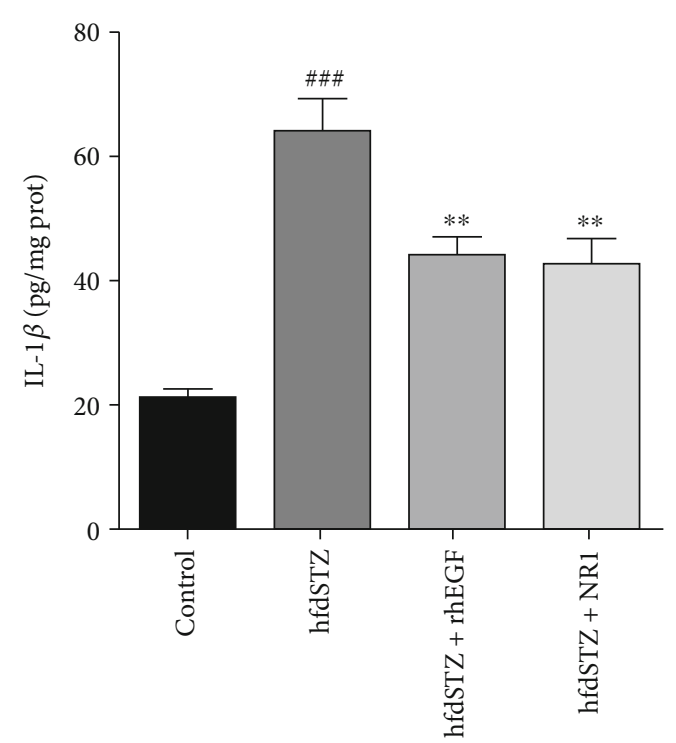

(c)

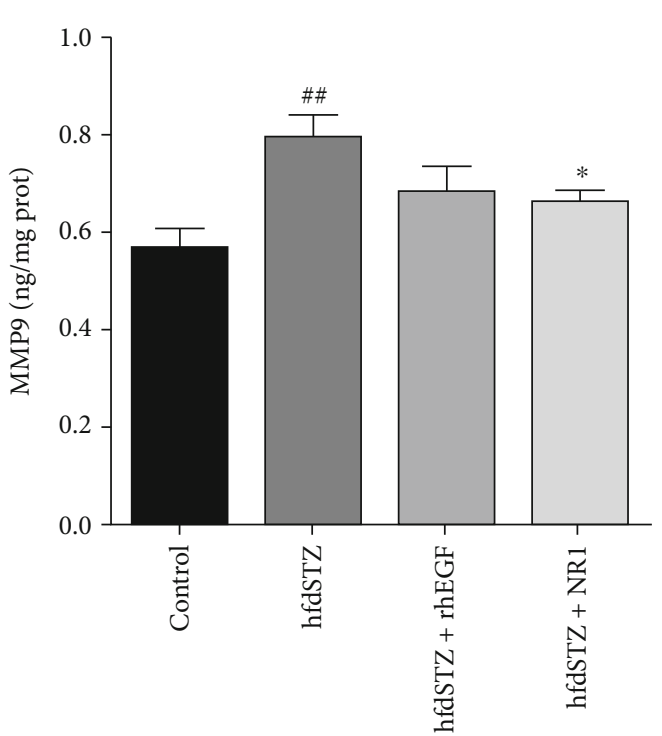

(b)

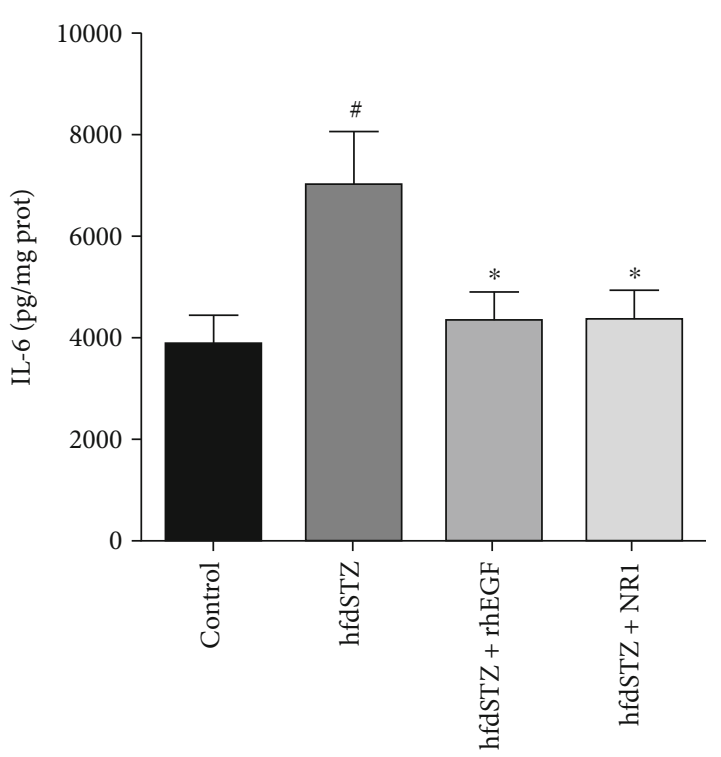

(d)

Figure 6: Continued. 


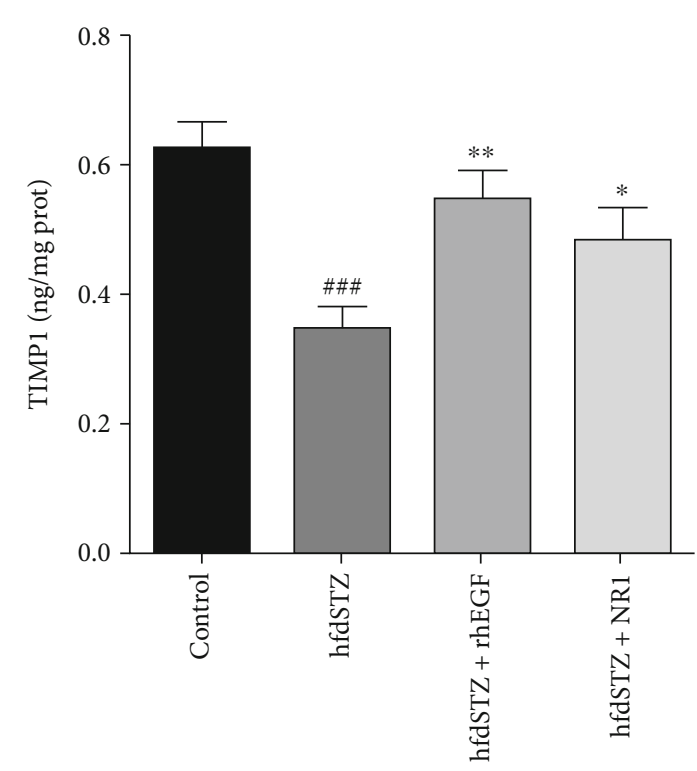

(e)

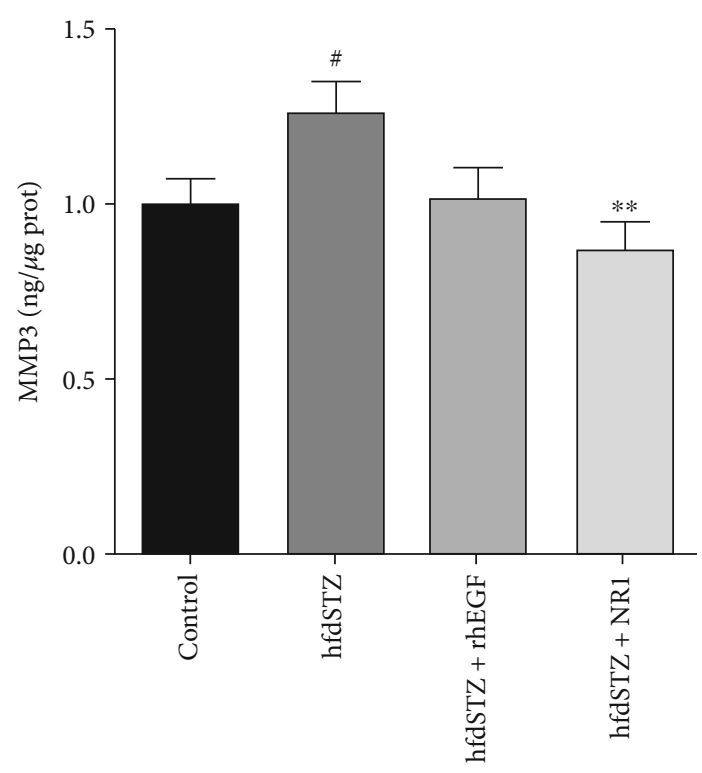

(f)

Figure 6: Effect of NR1 treatment on TGF- $\beta 1$, MMP9, IL-1 $\beta$, IL-6, TIMP1, and MMP3 in diabetic wounds. (a) ELISA results of TGF- $\beta 1$ $(n=8)$. (b) ELISA results of MMP9 $(n=8)$. (c) ELISA results of IL- $1 \beta(n=8)$. (d) ELISA results of IL-6 $(n=5-6)$. (e) ELISA results of TIMP1

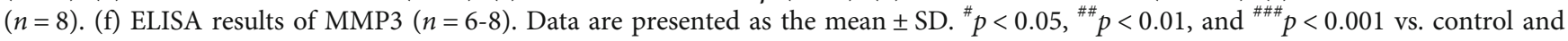
${ }^{*} p<0.05$ and ${ }^{* *} p<0.01$ vs. hfdSTZ.

MMP9 levels. In our study, we showed that topical NR1 treatment increased TGF- $\beta 1$ and TIMP1; decreased IL- $1 \beta$, IL-6, and MMP3, and MMP9; and contributed to enhanced ECM secretion and wound healing. It is difficult for diabetic wounds to heal spontaneously because of abnormal remodeling and impairment of ECM synthesis [48]. TGF- $\beta 1$ is a key regulator of the ECM in collagen synthesis $[49,50]$. In addition, the degradation of the ECM is regulated by tissue inhibitors of TIMPs and MMPs, both to maintain homeostasis of the ECM, and diabetic ulcer patients often demonstrate overproduction of MMPs with low TIMPs [51-53]. TIMP1 is a glycoprotein belonging to the family of TIMPs [54]; it inhibits most MMPs and may help perform its normal function $[55,56]$. Furthermore, TIMP1 also promotes cell proliferation and inhibits apoptosis [57]. A previous study found that increased MMP levels are associated with delayed wound healing [58]. MMP3 is a master controller of the matrix metalloproteinase family [59] and is able to activate a variety of matrix metalloproteinase precursors and participate in the degradation of the ECM and remodeling phases [60]. MMP9, a subtype of matrix metalloproteinase, is involved in the breakdown of extracellular matrix in normal physiological processes, such as tissue remodeling and metastasis [61]. These results demonstrated that the process of inflammation and the targets of TGF- $\beta 1$, TIMP1, MMP3, and MMP9 play a key role in the progression of NR1 treatment in diabetic ulcers.

\section{Conclusions}

In summary, NR1 has therapeutic efficacy against diabetic ulcers by reducing inflammation and apoptosis, increasing angiogenesis and maintaining ECM remodeling. Importantly, our study provides clear evidence for the successful use of topical NR1 treatment for type II diabetic ulcers, and its molecu- lar mechanism was explored. NR1 may have potential as a new, practical strategy for diabetic ulcer treatment.
Abbreviations
NR1: $\quad$ Notoginsenoside R1
T2DM: $\quad$ Type II diabetes mellitus
STZ: $\quad$ Streptozotocin
ECM: $\quad$ Extracellular matrix
CD31: $\quad$ Platelet endothelial cell adhesion molecule-1
TIMP1: Tissue inhibitor of metalloproteinase 1
TGF- $\beta 1$ : $\quad$ Transforming growth factor- $\beta 1$
MMP9: $\quad$ Matrix metallopeptidase 9
MMP3: $\quad$ Matrix metallopeptidase 9
IL-1 $\beta$ : Interleukin- $1 \beta$
IL-6: Interleukin-6
NPWT: Negative pressure wound therapy
EGF: $\quad$ Epidermal growth factor
TCM: Traditional Chinese medicine
TrxR1: Thioredoxin reductase 1
JNK: c-Jun N-terminal kinase
PNS: Panax Notoginseng Saponins
ET-1: Endothelin-1
TNF- $\alpha$ : Tumor necrosis factor- $\alpha$
RNA-Seq: RNA Sequencing
HE: Hematoxylin and eosin
DEs: Differentially expressed genes
ELISA: Enzyme-linked immunosorbent assay.

\section{Data Availability}

All data in this study can be obtained from the appropriate authors. 


\section{Conflicts of Interest}

The authors declare no conflicts of interest.

\section{Authors' Contributions}

G. Cao wrote the manuscript and performed animal experiments; J. Zhang, R. Zhou, C. Xiang, Y. Zhang, and H. Xu performed animal experiments, and C. Xiang performed the ELISA and immunohistochemistry; J. Zhang and $\mathrm{H}$. Yang conceived and supported this study; all authors reviewed and approved the final manuscript. Guangzhao Cao and Changpei Xiang contributed equally to this work.

\section{Acknowledgments}

The authors would like to acknowledge the financial support from the National Key R\&D Plan (No. 2017YFC1702500), Scientific and Technological Innovation Project of China Academy of Chinese Medical Sciences (CI2021A04612), National Natural Science Foundation of China (No. 81974550), and Fundamental Research Funds for the Central Public Welfare Research Institutes (Nos. ZZ13-YQ-046 and ZXKT21007).

\section{Supplementary Materials}

Supplementary 1. Tab. S1: composition of the experimental high-fat diet.

Supplementary 2. Tab. S2: gene expression of diabetic wounds treated with NR1 by using RNA-Seq analysis.

\section{References}

[1] G. Clerici and E. Faglia, "Saving the limb in diabetic patients with ischemic foot lesions complicated by acute infection," The International Journal of Lower Extremity Wounds, vol. 13, no. 4, pp. 273-293, 2014.

[2] J. Zhao, Y. Li, K. Deng, P. Yun, and T. Gong, "Therapeutic effects of static magnetic field on wound healing in diabetic rats," Journal of Diabetes Research, vol. 2017, Article ID 6305370, 5 pages, 2017.

[3] V. Falanga, "Wound healing and its impairment in the diabetic foot," The Lancet, vol. 366, no. 9498, pp. 1736-1743, 2005.

[4] F. M. Davis, A. Kimball, A. Boniakowski, and K. Gallagher, "Dysfunctional wound healing in diabetic foot ulcers: new crossroads," Current Diabetes Reports, vol. 18, no. 1, p. 2, 2018.

[5] A. K. Arya, R. Tripathi, S. Kumar, and K. Tripathi, "Recent advances on the association of apoptosis in chronic non healing diabetic wound," World Journal of Diabetes, vol. 5, no. 6, pp. 756-762, 2014.

[6] B. Kunkemoeller and T. R. Kyriakides, "Redox signaling in diabetic wound healing regulates extracellular matrix deposition," Antioxidants \& Redox Signaling, vol. 27, no. 12, pp. 823-838, 2017.

[7] A. E. Boniakowski, A. S. Kimball, B. N. Jacobs, S. L. Kunkel, and K. A. Gallagher, "Macrophage-mediated inflammation in normal and diabetic wound healing," The Journal of Immunology, vol. 199, no. 1, pp. 17-24, 2017.
[8] A. Shaikh-Kader, N. N. Houreld, N. K. Rajendran, and H. Abrahamse, "The link between advanced glycation end products and apoptosis in delayed wound healing," Cell Biochemistry and Function, vol. 37, no. 6, pp. 432-442, 2019.

[9] L. E. Tracy, R. A. Minasian, and E. J. Caterson, "Extracellular matrix and dermal fibroblast function in the healing wound," Advances in Wound Care, vol. 5, no. 3, pp. 119-136, 2016.

[10] R. Zhao, H. Liang, E. Clarke, C. Jackson, and M. Xue, "Inflammation in chronic wounds," International Journal of Molecular Sciences, vol. 17, no. 12, p. 2085, 2016.

[11] A. M. Boniakowski, A. D. denDekker, F. M. Davis et al., "SIRT3 regulates macrophage-mediated inflammation in diabetic wound repair," Journal of Investigative Dermatology, vol. 139, no. 12, pp. 2528-2537.e2, 2019.

[12] D. Liu, P. Yang, M. Gao et al., "NLRP3 activation induced by neutrophil extracellular traps sustains inflammatory response in the diabetic wound," Clinical Science, vol. 133, no. 4, pp. 565-582, 2019.

[13] W. Qi, C. Yang, Z. Dai et al., "High levels of pigment epithelium-derived factor in diabetes impair wound healing through suppression of Wnt signaling," Diabetes, vol. 64, no. 4, pp. 1407-1419, 2015.

[14] A. Jackson and S. Rajbhandari, "Developing a grapefruit model for assessment and training of diabetic foot ulcer debridement," Simulation in Healthcare: The Journal of the Society for Simulation in Healthcare, vol. 9, no. 5, pp. 331-336, 2014.

[15] Y. Liu, S. Zhou, Y. Gao, and Y. Zhai, "Electrospun nanofibers as a wound dressing for treating diabetic foot ulcer," Asian Journal of Pharmaceutical Sciences, vol. 14, no. 2, pp. 130143, 2019.

[16] L. A. Lavery, K. E. Davis, S. J. Berriman et al., "WHS guidelines update: diabetic foot ulcer treatment guidelines," Wound Repair and Regeneration, vol. 24, no. 1, pp. 112-126, 2016.

[17] T. Q. Bui, Q. V. P. Bui, D. Németh et al., "Epidermal growth factor is effective in the treatment of diabetic foot ulcers: meta-analysis and systematic review," International Journal of Environmental Research and Public Health, vol. 16, no. 14, p. 2584, 2019.

[18] Z. Ahmed, N. Husain, S. Nour, and S. H. Yee, "Efficacy of vacuum-assisted closure (VAC) in wound healing," Surgical Science, vol. 10, no. 6, pp. 173-215, 2019.

[19] S. Borys, J. Hohendorff, C. Frankfurter, B. Kiec-Wilk, and M. T. Malecki, "Negative pressure wound therapy use in diabetic foot syndrome-from mechanisms of action to clinical practice," European Journal of Clinical Investigation, vol. 49, no. 4, article e13067, 2019.

[20] F. Bassetto, L. Lancerotto, R. Salmaso et al., "Histological evolution of chronic wounds under negative pressure therapy," Journal of Plastic, Reconstructive \& Aesthetic Surgery, vol. 65, no. 1, pp. 91-99, 2012.

[21] J. Zhang, R. Zhou, C. Xiang, Q. Jia, H. Wu, and H. Yang, "Huangbai liniment accelerated wound healing by activating Nrf2 signaling in diabetes," Oxidative Medicine and Cellular Longevity, vol. 2020, Article ID 4951820, 20 pages, 2020.

[22] R. Zhou, C. Xiang, G. Cao et al., "Berberine accelerated wound healing by restoring TrxR1/JNK in diabetes," Clinical Science, vol. 135, no. 4, pp. 613-627, 2021.

[23] R. Uzayisenga, P. A. Ayeka, and Y. Wang, "Anti-diabetic potential of panax notoginseng saponins (PNS): a review," Phytotherapy Research, vol. 28, no. 4, pp. 510-516, 2014. 
[24] L. C. Yao, L. Wu, W. Wang et al., "Panax notoginseng saponins promote cell death and chemosensitivity in pancreatic cancer through the apoptosis and autophagy pathways," Anti-Cancer Agents in Medicinal Chemistry, vol. 21, no. 13, pp. 1680-1688, 2021.

[25] D. Zhou, K. Cen, W. Liu et al., "Xuesaitong exerts long-term neuroprotection for stroke recovery by inhibiting the ROCKII pathway, in vitro and in vivo," Journal of Ethnopharmacology, vol. 272, article 113943, 2021.

[26] Y. Yan, S. Li, C. Li et al., "Panax notoginsenoside saponins Rb1 regulates the expressions of Akt/ mTOR/PTEN signals in the hippocampus after focal cerebral ischemia in rats," Behavioural Brain Research, vol. 345, pp. 83-92, 2018.

[27] B. Zhang, X. Zhang, C. Zhang, Q. Shen, G. Sun, and X. Sun, "Notoginsenoside $\mathrm{R} 1$ protects $\mathrm{db} / \mathrm{db}$ mice against diabetic nephropathy via upregulation of Nrf2-mediated HO-1 expression," Molecules, vol. 24, no. 2, p. 247, 2019.

[28] Y. Zhai, X. Meng, Y. Luo et al., "Notoginsenoside R1 ameliorates diabetic encephalopathy by activating the Nrf2 pathway and inhibiting NLRP3 inflammasome activation," Oncotarget, vol. 9, no. 10, pp. 9344-9363, 2018.

[29] X. H. D. H. Yun-Guan, “X," Journal of Xinxiang Medical College, vol. 11, no. 29, pp. 815-817, 2012.

[30] Y. X. D. H. Yun-Guan, "Influence of Panax notoginsenoside on tumor necrosis factor- $\alpha$ in rats with diabetic skin ulceration," Journal of Xinxiang Medical College, vol. 12, no. 29, 2012.

[31] Y. Sun, H. Shi, S. Yin et al., "Human mesenchymal stem cell derived exosomes alleviate type 2 diabetes mellitus by reversing peripheral insulin resistance and relieving $\beta$-cell destruction," ACS Nano, vol. 12, no. 8, pp. 7613-7628, 2018.

[32] C. León, "Transcriptomic analysis of a diabetic skinhumanized mouse model dissects molecular pathways underlying the delayed wound healing response," Genes (Basel), vol. 12 , no. $1,2020$.

[33] S. Zhang, L. Chen, G. Zhang, and B. Zhang, "Umbilical cordmatrix stem cells induce the functional restoration of vascular endothelial cells and enhance skin wound healing in diabetic mice via the polarized macrophages," Stem Cell Research \& Therapy, vol. 11, no. 1, p. 39, 2020.

[34] Y. Guan, H. Niu, Z. Liu et al., "Sustained oxygenation accelerates diabetic wound healing by promoting epithelialization and angiogenesis and decreasing inflammation," Science Advances, vol. 7, no. 35, 2021.

[35] K. Chaturvedi and D. K. Sarkar, "Isolation and characterization of rat pituitary endothelial cells," Neuroendocrinology, vol. 83, no. 5-6, pp. 387-393, 2006.

[36] Z. F. Han, J. H. Cao, Z. Y. Liu, Z. Yang, R. X. Qi, and H. L. Xu, "Exosomal lncRNA KLF3-AS1 derived from bone marrow mesenchymal stem cells stimulates angiogenesis to promote diabetic cutaneous wound healing," DIABETES RESEARCH AND CLINICAL PRACTICE, vol. 183, article 109126, 2022.

[37] A. Desmouliere, M. Redard, I. Darby, and G. Gabbiani, “Apoptosis mediates the decrease in cellularity during the transition between granulation tissue and scar," American Journal of Pathology, vol. 146, no. 1, pp. 56-66, 1995.

[38] L. Yang, L. Lei, Q. Zhao, Y. Gong, G. Guan, and S. Huang, "Ctype natriuretic peptide/natriuretic peptide receptor 2 is involved in cell proliferation and testosterone production in mouse Leydig cells," World Journal of Mens Health, vol. 37, no. 2, pp. 186-198, 2019.
[39] M. F. Brown, B. J. Leibowitz, D. Chen et al., "Loss of_Caspase3_ sensitizes colon cancer cells to genotoxic stress via RIP1dependent necrosis," Cell Death \& Disease, vol. 6, no. 4, article e1729, 2015.

[40] P. Wang, Y. Zhao, J. Wang et al., "Dephosphorylation of caveolin-1 controls C-X-C motif chemokine ligand 10 secretion in mesenchymal stem cells to regulate the process of wound healing," Frontiers in cell and developmental biology, vol. 9, article 725630, 2021.

[41] J. A. van Dongen, V. Getova, L. A. Brouwer et al., "Adipose tissue-derived extracellular matrix hydrogels as a release platform for secreted paracrine factors," Journal of Tissue Engineering and Regenerative Medicine, vol. 13, no. 6, pp. 973-985, 2019.

[42] M. Coates, S. Blanchard, and A. S. Macleod, "Innate antimicrobial immunity in the skin: a protective barrier against bacteria, viruses, and fungi," PLoS Pathogens, vol. 14, no. 12, article e1007353, 2018.

[43] R. E. Mirza, M. M. Fang, E. M. Weinheimer-Haus, W. J. Ennis, and T. J. Koh, "Sustained inflammasome activity in macrophages impairs wound healing in type 2 diabetic humans and mice," Diabetes, vol. 63, no. 3, pp. 1103-1114, 2014.

[44] K. Imajo, M. Yoneda, T. Kessoku et al., "Rodent models of nonalcoholic fatty liver disease/nonalcoholic steatohepatitis," International Journal of Molecular Sciences, vol. 14, no. 11, pp. 21833-21857, 2013.

[45] X. Zhao, S. Li, J. Ding et al., "Combination of an engineered Lactococcus lactis expressing CXCL12 with light-emitting diode yellow light as a treatment for scalded skin in mice," Microbial Biotechnology, vol. 14, no. 5, pp. 2090-2100, 2021.

[46] X. Sun, X. Wang, Z. Zhao, J. Chen, C. Li, and G. Zhao, "Paeoniflorin inhibited nod-like receptor protein-3 inflammasome and NF- $\kappa \mathrm{B}$-mediated inflammatory reactions in diabetic foot ulcer by inhibiting the chemokine receptor CXCR2," Drug Development Research, vol. 82, no. 3, pp. 404-411, 2021.

[47] K. Muller and V. Meineke, "Radiation-induced alterations in cytokine production by skin cells," Experimental Hematology, vol. 35, no. 4, pp. 96-104, 2007.

[48] H. Chen, Y. Peng, S. Wu, and L. P. Tan, "Electrospun 3D fibrous scaffolds for chronic wound repair," Materials (Basel), vol. 9, no. 4, p. 272, 2016.

[49] X. Ma, C. Lu, C. Lv, C. Wu, and Q. Wang, "The expression of miR-192 and its significance in diabetic nephropathy patients with different urine albumin creatinine ratio," Journal of Diabetes Research, vol. 2016, Article ID 6789402, 6 pages, 2016.

[50] N. A. Alhakamy, G. Caruso, B. G. Eid et al., "Ceftriaxone and melittin synergistically promote wound healing in diabetic rats," Pharmaceutics, vol. 13, no. 10, p. 1622, 2021.

[51] M. Muller, C. Trocme, B. Lardy, F. Morel, S. Halimi, and P. Y. Benhamou, "Matrix metalloproteinases and diabetic foot ulcers: the ratio of MMP-1 to TIMP-1 is a predictor of wound healing," Diabetic Medicine, vol. 25, no. 4, pp. 419-426, 2008.

[52] D. M. Jaworski, P. Soloway, J. Caterina, and W. A. Falls, “Tissue inhibitor of metalloproteinase-2(TIMP-2)-deficient mice display motor deficits," Journal of Neurobiology, vol. 66, no. 1, pp. 82-94, 2006.

[53] J. Pei, M. Harakalova, T. A. Treibel et al., "H3K27ac acetylome signatures reveal the epigenomic reorganization in remodeled non-failing human hearts," Clinical Epigenetics, vol. 12, no. 1, p. 106, 2020. 
[54] J. R. Allen, L. Ge, Y. Huang et al., "TIMP-1 promotes the immune response in influenza-induced acute lung injury," Lung, vol. 196, no. 6, pp. 737-743, 2018.

[55] M. Egeblad and Z. Werb, "New functions for the matrix metalloproteinases in cancer progression," Nature Reviews Cancer, vol. 2, no. 3, pp. 161-174, 2002.

[56] C. R. Ban and S. M. Twigg, "Fibrosis in diabetes complications: pathogenic mechanisms and circulating and urinary markers," Vascular Health and Risk Management, vol. 4, no. 3, pp. 575596, 2008.

[57] B. Grunwald, B. Schoeps, and A. Kruger, "Recognizing the molecular multifunctionality and interactome of TIMP-1," Trends in Cell Biology, vol. 29, no. 1, pp. 6-19, 2019.

[58] A. Tardaguila-Garcia, E. Garcia-Morales, J. M. Garcia-Alamino, F. J. Alvaro-Afonso, R. J. Molines-Barroso, and J. L. LazaroMartinez, "Metalloproteinases in chronic and acute wounds: a systematic review and meta-analysis," Wound Repair and Regeneration, vol. 27, no. 4, pp. 415-420, 2019.

[59] M. Affara, B. J. Dunmore, D. A. Sanders, N. Johnson, C. G. Print, and D. S. Charnock-Jones, "MMP1 bimodal expression and differential response to inflammatory mediators is linked to promoter polymorphisms," BMC Genomics, vol. 12, no. 1, p. 43, 2011.

[60] R. Zamborsky, M. Kokavec, S. Harsanyi, and L. Danisovic, "Identification of prognostic and predictive osteosarcoma biomarkers," Medical Sciences, vol. 7, no. 2, p. 28, 2019.

[61] M. Zaleska, W. L. Olszewski, M. Durlik, and N. E. Miller, "Signaling proteins are represented in tissue fluid/lymph from soft tissues of normal human legs at concentrations different from serum," Lymphatic Research and Biology, vol. 11, no. 4, pp. 203-210, 2013. 